\title{
How to Derotate the Cosmic Microwave Background Polarization
}

\begin{abstract}
Marc Kamionkowski
California Institute of Technology, Mail Code 130-33, Pasadena, California 91125, USA

(Received 7 October 2008; revised manuscript received 12 February 2009; published 19 March 2009)

If the linear polarization of the cosmic microwave background is rotated in a frequency-independent manner as it propagates from the surface of last scatter, it may introduce a $B$-mode polarization. Here I show that measurement of higher-order $T E, E E, E B$, and $T B$ correlations induced by this rotation can be used to reconstruct the rotation angle as a function of position on the sky. This technique can be used to distinguish primordial $B$ modes from those induced by rotation. The effects of rotation can be distinguished geometrically from similar effects due to cosmic shear.
\end{abstract}

DOI: 10.1103/PhysRevLett.102.111302

PACS numbers: $98.70 . V \mathrm{c}$

The polarization of the cosmic microwave background $(\mathrm{CMB})$ can be decomposed into gradient ( $E$ mode) and curl ( $B$ mode) components [1]. Primordial density perturbations produce a polarization pattern that is purely $E$ mode at the surface of last scatter, while primordial gravitational waves, such as those from inflation, produce a $B$ mode [2]. There is now an active experimental effort to detect $B$ modes, as these would constitute a "smoking gun" for inflationary gravitational waves [3].

There may, however, be other mechanisms - apart from gravitational waves-for producing $B$ modes. The most widely considered is cosmic shear: The deflection of CMB photons due to weak gravitational lensing by density perturbations along the line of sight will convert some of the $E$ mode at the surface of last scatter to a $B$ mode [4]. Cosmic shear of the CMB is no longer the realm of futuristic theorists; it has recently been detected [5].

Another possibility is a rotation of the linear polarization of the CMB as it travels from the surface of last scatter [6]. This could occur, for example, if there is a quintessence field that couples to the pseudoscalar of electromagnetism [7]. In this case, the polarization would be rotated by an angle $\alpha$ that is uniform across the sky. A fraction $\sin 2 \alpha$ of the $E$ mode would thus be converted into a $B$ mode [6]. This $B$ mode could be distinguished from that due to gravitational waves by the parity-breaking $E B$ and $T B$ cross correlations that it produces, as well as by the shape of the $T B, E B$, and $B B$ power spectra. Searches with current data already constrain such a uniform rotation to be no more than a few degrees $[8,9]$.

But what if the rotation angle $\alpha$ varies across the sky? The authors in Ref. [10] have recently proposed models in which this might occur by virtue of a spatially and timevarying scalar field coupled to the pseudoscalar of electromagnetism, and something similar may arise from darkmatter magnetic moments [11]. (Faraday rotation could also rotate the polarization [12], but this can be identified with multifrequency maps.) If the distribution of rotation angles is symmetric about zero, then there will be a $B$ mode induced with no parity-breaking $T B$ or $E B$ correlations
[10]. Can this type of $B$ mode be distinguished from that due to primordial gravitational waves?

In this Letter, I show that a position-dependent rotation of the polarization induces higher-order correlations in the temperature polarization of the CMB. I then show how these new correlations can be used to measure the rotation angle $\alpha(\hat{\boldsymbol{n}})$ as a function of position $\hat{\boldsymbol{n}}$ on the sky. The observed polarization pattern can then be corrected to construct the primordial polarization pattern. It will thus be possible to distinguish whether a $B$-mode pattern, if detected, is primordial or due to a post-recombination rotation. The techniques I will discuss can also be used to test an experiment for systematic artifacts.

The reconstruction algorithm will be similar to that developed to reconstruct the cosmic-shear field [13] and also to that developed to test for statistical isotropy (SI) [14]. In fact, the effects of rotation may be viewed as a possible contaminant for cosmic-shear maps. However, the effects of cosmic shear (or SI violations) and rotation can be distinguished, as (a) rotation has a different geometric effect on the polarization and (b) cosmic shear affects the temperature as well.

The linear polarization at each point $\hat{\boldsymbol{n}}$ on the sky is quantified by Stokes parameters $Q(\hat{\boldsymbol{n}})$ and $U(\hat{\boldsymbol{n}})$, measured with respect to the $\hat{\theta}-\hat{\phi}$ axes. These Stokes parameters are components of a symmetric trace-free $2 \times 2$ polarization tensor field $\mathcal{P}_{a b}(\hat{\boldsymbol{n}})$ which can be expanded in terms of tensor spherical harmonics as

$$
\mathcal{P}_{a b}(\hat{\boldsymbol{n}})=\sum_{l=2}^{\infty} \sum_{m=-l}^{l}\left[E_{l m} Y_{(l m) a b}^{E}(\hat{\boldsymbol{n}})+B_{l m} Y_{(l m) a b}^{B}(\hat{\boldsymbol{n}})\right]
$$

Here $Y_{(l m) a b}^{E}(\hat{\boldsymbol{n}})$ and $Y_{(l m) a b}^{B}(\hat{\boldsymbol{n}})$ are complete sets of basis functions for the gradient ( $E$ mode) and curl ( $B$ mode) components of the polarization. Orthonormality of the basis functions allows us to write the expansion coefficients as

$$
E_{l m}=\int d \hat{\boldsymbol{n}} \mathcal{P}_{a b}(\hat{\boldsymbol{n}}) Y_{(l m)}^{E a b *}(\hat{\boldsymbol{n}}),
$$




$$
B_{l m}=\int d \hat{\boldsymbol{n}} \mathcal{P}_{a b}(\hat{\boldsymbol{n}}) Y_{(l m)}^{B a b *}(\hat{\boldsymbol{n}}) .
$$

Suppose now that the polarization pattern at the surface of last scatter has no $B$ mode and that the polarization at each point $\hat{\boldsymbol{n}}$ is rotated by an angle $\alpha(\hat{\boldsymbol{n}})$. In that case, the observed Stokes parameters will be

$$
\begin{aligned}
\left(\begin{array}{c}
Q_{\text {obs }}(\hat{\boldsymbol{n}}) \\
U_{\text {obs }}(\hat{\boldsymbol{n}})
\end{array}\right) & =\left(\begin{array}{cc}
\cos 2 \alpha(\hat{\boldsymbol{n}}) & \sin 2 \alpha(\hat{\boldsymbol{n}}) \\
-\sin 2 \alpha(\hat{\boldsymbol{n}}) & \cos 2 \alpha(\hat{\boldsymbol{n}})
\end{array}\right)\left(\begin{array}{l}
Q(\hat{\boldsymbol{n}}) \\
U(\hat{\boldsymbol{n}})
\end{array}\right) \\
& =\cos 2 \alpha\left(\begin{array}{c}
Q \\
U
\end{array}\right)+\sin 2 \alpha\left(\begin{array}{c}
U \\
-Q
\end{array}\right) .
\end{aligned}
$$

The concordance of measured $T T, T E$, and $E E$ power spectra suggests that $\alpha$ is relatively small and that the uniform component of $\alpha$ is small [8]. We therefore work to linear order in $\alpha$. The change to the polarization tensor induced by the rotation is then $\delta \mathcal{P}_{a b}(\hat{\boldsymbol{n}})=2 \alpha(\hat{\boldsymbol{n}}) \mathcal{P}_{a b}^{r}(\hat{\boldsymbol{n}})$, where $\mathcal{P}_{a b}^{r}(\hat{\boldsymbol{n}})$ is rotated from the original polarization $\mathcal{P}_{a b}$ by $45^{\circ}$. Thus, if $\mathcal{P}_{a b}$ is pure $E$ mode, then $\mathcal{P}_{a b}^{r}$ is pure $B$ mode and can be written as

$$
\mathcal{P}_{a b}^{r}(\hat{\boldsymbol{n}})=\sum_{l=2}^{\infty} \sum_{m=-l}^{l} E_{l m} Y_{(l m) a b}^{B} .
$$

To calculate the curl component induced by rotation, we insert $\delta \mathcal{P}_{a b}$ into the expression [Eq. (3)] for $B_{l m}$. We then expand the rotation angle in terms of (scalar) spherical harmonics $Y_{(I m)}$ as $\alpha(\hat{\boldsymbol{n}})=\sum_{L M} \alpha_{L M} Y_{(L M)}(\hat{\boldsymbol{n}})$, to obtain

$$
\begin{aligned}
B_{l m} & =\sum_{L M} 2 \sum_{l_{2} m_{2}} \alpha_{L M} E_{l_{2} m_{2}} \int d \hat{\boldsymbol{n}} Y_{(l m)}^{B, a b *} Y_{(L M)} Y_{\left(l_{2} m_{2}\right) a b}^{B} \\
& =2(-1)^{m} \sum_{L M} \sum_{l_{2} m_{2}} \alpha_{L M} E_{l_{2} m_{2}} \xi_{l m l_{2} m_{2}}^{L M} H_{l l_{2}}^{L},
\end{aligned}
$$

where the sum is taken only over $l_{2}$ values that satisfy $L+$ $l+l_{2}=$ even,

$$
H_{l l^{\prime}}^{L} \equiv\left(\begin{array}{ccc}
l & l^{\prime} & L \\
-2 & 2 & 0
\end{array}\right)\left(\begin{array}{ccc}
l & l^{\prime} & L \\
0 & 0 & 0
\end{array}\right)^{-1}
$$

in terms of Wigner- $3 j$ symbols, and

$$
\xi_{l m l^{\prime} m^{\prime}}^{L M}=\int d \hat{\boldsymbol{n}} Y_{(l m)}^{*}(\hat{\boldsymbol{n}}) Y_{\left(l^{\prime} m^{\prime}\right)}(\hat{\boldsymbol{n}}) Y_{(L M)}(\hat{\boldsymbol{n}}) .
$$

We thus see that rotation induces a $B$ mode $[6,10]$. If there is a power spectrum for $\alpha_{L M}$, then the CMB power spectrum $C_{l}^{B B}$ can be calculated from Eq. (6) [10].

There is also an $O(\alpha)$ change in the $E$ mode induced by rotation with precisely the same form as Eq. (6) but with contributions only from $L+l+l_{2}=$ odd, rather than even. In the discussion below, we focus for brevity and clarity on the induced $E B$ and $T B$ correlations. However, the entire discussion applies (with differences to be pointed out below) to induced $E E$ and $T E$ correlations.
The next step is to consider the correlation of the induced $B$ mode with the original $E$ mode, as well as with the temperature $T$. Recalling that the original $E$ modes have (assuming Gaussian initial conditions) expectation values $\left\langle E_{l m}^{*} E_{l^{\prime} m^{\prime}}\right\rangle=C_{l}^{E E} \delta_{l l^{\prime}} \delta_{m m^{\prime}}$ (where $C_{l}^{E E}$ is the $E E$ power spectrum), the correlation between the observed $E$ and $B$ modes is [15]

$$
\begin{aligned}
\left\langle B_{l m} E_{l^{\prime} m^{\prime}}^{*}\right\rangle= & 2 \frac{\alpha_{00}}{\sqrt{4 \pi}} C_{l}^{E E} \delta_{l l^{\prime}} \delta_{m m^{\prime}} \\
& +2 \sum_{L \geq 1} \sum_{M=-L}^{L} \alpha_{L M} C_{l}^{E E} \xi_{l m l^{\prime} m^{\prime}}^{L M} H_{l l^{\prime}}^{L}
\end{aligned}
$$

I have split off the $L=0$ term in Eq. (9) to show that the result for a uniform rotation angle $\alpha$ is $\left\langle B_{l m} E_{l^{\prime} m^{\prime}}^{*}\right\rangle=$ $2 \alpha C_{l}^{E E} \delta_{l l^{\prime}} \delta_{m m^{\prime}}$, as it should be [6]. The sum in Eq. (9) is taken only over $L+l+l^{\prime}=$ even. The expression for the induced correlations $\left\langle E_{l m} E_{l^{\prime} m^{\prime}}^{*}\right\rangle$ is the same as that in Eq. (9) but with $L+l+l^{\prime}=$ odd and no $L=0$ contribution.

Likewise, given that the temperature $T$ and the $E$-mode polarization are correlated at the surface of last scatter, there will also be a nonzero $T B$ correlation induced by rotation. The expression for $\left\langle T_{l m} B_{l^{\prime} m^{\prime}}^{*}\right\rangle$ is identical to Eq. (9) with the replacement $C_{l}^{E E} \rightarrow C_{l}^{T E}$, again for $L+$ $l+l^{\prime}=$ even. There will also be $T E$ correlations of the same form but with $L+l+l^{\prime}=$ odd and no $L=0$ term.

If $\alpha(\hat{\boldsymbol{n}})$ varies with $\hat{\boldsymbol{n}}$, then $\alpha_{L M} \neq 0$ for $L \geq 1$, and if so, there will be correlations between $E_{l m}$ and $B_{l^{\prime} m^{\prime}}$ (and $T_{l m}$ and $B_{l^{\prime} m^{\prime}}$ ) of different $l m$ and $l^{\prime} m^{\prime}$. The existence of these off-diagonal correlations can be used to measure each of the rotation multipole moments $\alpha_{l m}$ and thus $\alpha(\hat{\boldsymbol{n}})$. The relevant formalism is similar to that for measuring the cosmic-shear field [13] or for searching for SI violations [14], so we can adopt results from prior work. To do so, we note that Eq. (9) is identical to Eq. (A1) in Ref. [14] with the identification $X=B$ (or $T$ in place of $B$ ), $X^{\prime}=E$, and $D_{l l^{\prime}}^{L M, X X^{\prime}}=\alpha_{L M} C_{l}^{E E} H_{l l^{\prime}}^{L}$ (or $C_{l}^{T E}$ in place of $C_{l}^{E E}$ for $T B$ ).

Our goal is to obtain the minimum-variance estimator $\hat{\alpha}_{L M}$ that can be obtained from a full-sky polarization map or a temperature-polarization map. We suppose that the maps are provided as a measured temperature $T^{\operatorname{map}}(\hat{\boldsymbol{n}})$ and Stokes parameters $Q^{\operatorname{map}}(\hat{\boldsymbol{n}})$ and $U^{\operatorname{map}}(\hat{\boldsymbol{n}})$ in $N_{\text {pix }}$ pixels on the sky. The temperature (polarization) in each pixel receives contributions from the signal, which is the temperature (polarization) on the sky smoothed with a Gaussian beam of full width at half maximum (FWHM) $\theta_{\text {fwhm }}$, and a Gaussian noise with variance $\sigma_{T}^{2}\left(\sigma_{P}^{2}\right)$. The power spectra for the map are then $C_{l}^{A \text {,map }}=\left|W_{l}^{2}\right| C_{l}^{A}+C_{l}^{A, n}$, where $C_{l}^{A, n}$ is the noise power spectrum for $A$ (e.g., $A=$ $\{T T, E E, B B, T E, T B, E B\})$. These are $C_{l}^{T T, n}=$ $\left(4 \pi / N_{\text {pix }}\right) \sigma_{T}^{2}, \quad C_{l}^{E E, n}=C_{l}^{B B, n}=\left(4 \pi / N_{\text {pix }}\right) \sigma_{P}^{2}, \quad$ and $C_{l}^{T E, n}=C_{l}^{T B, n}=C_{l}^{E B, n}=0$. Beam smearing is taken into 
account with the window function $W_{l}=\exp \left(-l^{2} \sigma_{b}^{2} / 2\right)$, with $\sigma_{b}=\theta_{\text {fwhm }} / \sqrt{8 \ln 2}=0.0742\left(\theta_{\text {fwhm }} / 1^{\circ}\right)$.

We now derive the minimum-variance estimator $\hat{\alpha}_{L M}$ that can be obtained from $E B$ correlations; the results for the estimator that can be obtained from $T B$ correlations will be identical with the replacement $E \rightarrow B ; T E$ and $E E$ estimators are similarly derived.

By following Ref. [14], the minimum-variance estimator for each $D_{l l^{\prime}}^{L M}=2 \alpha_{L M} C_{l}^{E E} H_{l l^{\prime}}^{L}$ that can be obtained from the polarization map is (see also Ref. [16])

$$
\hat{D}_{l l^{\prime}}^{L M, \text { map }}=\left(G_{l l^{\prime}}^{L}\right)^{-1} \sum_{m m^{\prime}} B_{l m}^{\operatorname{map}}\left(E_{l^{\prime} m^{\prime}}^{\mathrm{map}}\right)^{*} \xi_{l m l^{\prime} m^{\prime}}^{L M}
$$

with

$$
4 \pi(2 L+1) G_{l l^{\prime}}^{L}=(2 l+1)\left(2 l^{\prime}+1\right)\left(C_{l 0 l^{\prime} 0}^{L 0}\right)^{2},
$$

where $C_{l m l^{\prime} m^{\prime}}^{L M}$ is a Clebsch-Gordan coefficient.

The coefficient $\alpha_{L M}$ can be estimated from measurement of $D_{l l^{\prime}}^{L M}$ from each $l l^{\prime}$ pair through $\alpha_{L M}=$ $D_{l l^{\prime}}^{L M} /\left(2 C_{l}^{E E} H_{l l^{\prime}}^{L}\right)$. One can then average the estimates of $\alpha_{l l^{\prime}}$ from all of the $l l^{\prime}$ pairs. The trick, though, is to weight these all in a manner that minimizes the variance to $\alpha_{L M}$. If each estimator $\hat{D}_{l l^{\prime}}^{L M \text {,map }}$ were statistically independent, then we could simply weight by the inverse variance. However, things are a bit (though not much) more complicated.

Each of the estimators $\hat{D}_{l l^{\prime}}^{L M \text {,map }}$ are statistically independent for different $L M$. They are also statistically independent for different $l l^{\prime}$, except that $\hat{D}_{l l^{\prime}}^{L M \text {, map }}$ is correlated with $\hat{D}_{l^{\prime} l}^{L M \text {,map }}$. To take this into account, we take $l^{\prime} \geq l$ and then consider for $l \neq l^{\prime} E B$ modes as well as $B E$ modes. For $l=$ $l^{\prime}$, there is only a single variance; for $l^{\prime}>l$, there is a $2 \times 2$ covariance matrix in the $E B-B E$ space.

Write the covariances between the different $\hat{D}_{l l^{\prime}}^{L M \text {,map }}$ as

$$
\mathcal{C}_{A A^{\prime}}^{l l^{\prime}} \equiv G_{l l^{\prime}}^{L}\left\langle\hat{D}_{l l^{\prime}}^{L M, A, \text { map }} \hat{D}_{l l^{\prime}}^{L M, A^{\prime}, \text { map }}\right\rangle,
$$

for $\left\{A, A^{\prime}\right\}=\{E B, B E\}$. For $l=l^{\prime}$, there is no distinction between $E B$ and $B E$; the variance in this case is then $\mathcal{C}_{B E, B E}^{l l}=\frac{1}{2}\left[C_{l}^{B B \text {,map }} C_{l}^{E E \text {,map }}\right]$. For $l^{\prime}>l$, the covariances are $\mathcal{C}_{B E, B E}^{l l^{\prime}}=C_{l}^{B B \text {,map }} C_{l^{\prime}}^{E E \text {,map }}, \mathcal{C}_{E B, E B}^{l l^{\prime}}=C_{l^{\prime}}^{B B \text {,map }} C_{l}^{E E \text {,map }}$, and $\mathcal{C}_{B E, E B}^{l l^{\prime}}=C_{l}^{B E \text {,map }} C_{l^{\prime}}^{B E \text { map }}$.

We now write two estimators $\hat{\alpha}_{L M}^{l=l^{\prime}}$ and $\hat{\alpha}_{L M}^{l<l^{\prime}}$, the first coming from $E B$ correlations with $l=l^{\prime}$ and the second from those with $l<l^{\prime}$. We will then average them, with inverse-variance weighting, to obtain the final estimator. The first is

$$
\hat{\alpha}_{L M}^{l=l^{\prime}}=\frac{\sum_{l} F_{l l}^{L}\left(W_{l}\right)^{2} \hat{D}_{l l}^{L M, \text { map }} G_{l l}^{L} / \mathcal{C}_{B E, B E}^{l l}}{\sum_{l}\left[F_{l l}^{L}\left(W_{l}\right)^{2}\right]^{2} G_{l l}^{L} / \mathcal{C}_{B E, B E}^{l l}}
$$

where $F_{l l^{\prime}}^{L} \equiv 2 C_{l}^{E E} H_{l l^{\prime}}^{L}$. The second is

$$
\hat{\alpha}_{L M}^{l<l^{\prime}}=\frac{\sum_{l^{\prime}>l} W_{l} W_{l^{\prime}} G_{l l^{\prime}}^{L} F_{l l^{\prime}}^{L} \sum_{A A^{\prime}} \hat{D}_{l l^{\prime}}^{L M, A, \operatorname{map}}\left[\left(\mathcal{C}^{l l}\right)^{-1}\right]_{A A^{\prime}}}{\sum_{l^{\prime}>l}\left(W_{l} W_{l^{\prime}}\right)^{2} G_{l l^{\prime}}^{L} F_{l l^{\prime}}^{L} F_{l^{\prime} l}^{L} \sum_{A A^{\prime}}\left[\left(\mathcal{C}^{l l}\right)^{-1}\right]_{A A^{\prime}}},
$$

where the matrix inversion is in the $2 \times 2 E B-B E$ basis. Note that the superscripts $A$ on $\hat{D}$ are necessary in these expressions, as these quantities differ for $E B$ and $B E$. The variance to the first estimator is

$$
\left(\sigma_{\alpha_{L M}^{l=l^{\prime}}}\right)^{-2}=\sum_{l} \frac{\left[F_{l l}^{L}\left(W_{l}\right)^{2}\right]^{2} G_{l l}^{L}}{\mathcal{C}_{B E, B E}^{l l}}
$$

The variance $\left(\sigma_{\alpha_{L M}^{l<l^{\prime}}}\right)^{2}$ to the second estimator is obtained from

$$
\left(\sigma_{\alpha_{L M}^{l<l^{\prime}}}\right)^{-2}=\sum_{l^{\prime}>l} G_{l l^{\prime}}^{L} F_{l^{\prime} l}^{L} F_{l l^{\prime}}^{L}\left(W_{l} W_{l^{\prime}}\right)^{2} \sum_{A A^{\prime}}\left[\left(\mathcal{C}^{l l^{\prime}}\right)^{-1}\right]_{A A^{\prime}}
$$

The final minimum-variance estimator $\hat{\alpha}_{L M}$ is then obtained by averaging, with inverse-variance weighting, the two estimators above:

$$
\hat{\alpha}_{L M}=\frac{\hat{\alpha}_{L M}^{l=l^{\prime}}\left(\sigma_{\alpha_{L M}^{l=l^{\prime}}}\right)^{-2}+\hat{\alpha}_{L M}^{l<l^{\prime}}\left(\sigma_{\alpha_{L M}^{l<l^{\prime}}}\right)^{-2}}{\left(\sigma_{\alpha_{L M}^{l=l^{\prime}}}\right)^{-2}+\left(\sigma_{\alpha_{L M}^{l<l^{\prime}}}\right)^{-2}} .
$$

The variance $\left(\sigma_{\alpha_{L M}}\right)^{2}$ for this estimator is given by $\left(\sigma_{\alpha_{L M}}\right)^{-2}=\left(\sigma_{\alpha_{L M}^{l=l^{\prime}}}\right)^{-2}+\left(\sigma_{\alpha_{L M}^{l<l^{\prime}}}\right)^{-2}$. For small $L$ (e.g., a rotation dipole), the two terms will contribute comparably to the statistical weight. For larger $L$, the $l^{\prime}>l$ estimator should carry most of the statistical weight.

Estimators from $T B$ correlations are identical to the $E B$ estimators discussed above with the replacement $E \rightarrow B$. Likewise, there will be $E E$ and $T E$ correlations similarly induced. (Things simplify for $E E$, as the $2 \times 2 A A^{\prime}$ covariance matrix becomes a single variance.) The estimators for $\alpha_{L M}$ for $E E$ and $T E$ and their variances can be constructed analogously. There will also be $B B$ correlations, but they will be higher order in $\alpha$. There will be no TT correlations induced, as the rotation does not act on the temperature. Since $T$ and $E$ are correlated in the primordial polarization field, there will be cross correlations between the estimators $\hat{\alpha}_{L M}$ from $E B, T B, E E$, and $T E$. This may be an orderunity effect if the statistical weights of the various estimators are comparable. The expressions for the complete covariances are long, and so I leave them for future work.

Here I have shown that CMB temperature-polarization statistics can be developed to measure the angle $\alpha(\hat{\boldsymbol{n}})$ by which CMB photons were rotated, en route from the surface of last scatter, as a function of position $\hat{\boldsymbol{n}}$ on the sky. Explicit formulas to obtain the coefficients $\alpha_{L M}$ in a spherical-harmonic expansion of $\alpha(\hat{\boldsymbol{n}})$ from a full-sky CMB map were provided. This technique can then deter- 
mine whether $B$ modes, if detected, occur at the surface of last scatter or are due to a post-recombination rotation of the polarization. It is interesting to know that the rotation angle can be determined from the data, rather than by assumption. And if the rotation angle is assumed to be zero, then the techniques developed here can provide a test for systematic artifacts in the data. (Reference [17] suggested tests for systematics along these lines.)

The rotation and cosmic-shear formalisms share some similarities, and so rotation, if it exists, could show up as an artifact in a cosmic-shear analysis. However, the detailed effects are different and can be distinguished in the data. First of all, cosmic shear has a different parity than rotation; a given $L$ mode of the cosmic-shear field correlates $l$ and $l^{\prime}$ modes of $E$ and $B$, respectively, only if $l+l^{\prime}+L$ is odd, while rotation correlates them only for $l+l^{\prime}+L$ odd. Furthermore, cosmic shear acts on temperature and polarization, while rotation leaves the temperature map unaltered.

The formulas for the estimators $\hat{\alpha}_{L M}$ will need to be modified to take into account partial-sky coverage in a realistic map. However, it will be straightforward to adapt the techniques that have been developed to measure cosmic shear of the CMB on a partial sky to measure the rotation angle. Likewise, it is straightforward to simplify the fullsky analysis performed here to the flat-sky limit, which may be appropriate for suborbital experiments that map the $\mathrm{CMB}$ on a small patch of sky. Since the formalism to reconstruct the rotation angle resembles that to determine the cosmic-shear field, there may be other cosmic-shear techniques that can be adapted for rotation. For example, maximum-likelihood techniques [18] may be developed to provide even more sensitive probes of rotation than the quadratic estimators discussed here.

Finally, it is of interest to know quantitatively how well the estimators presented here can be used to construct the rotation angle. Evaluating the expressions for $\sigma_{\alpha_{L M}}$ for Wilkinson Microwave Anisotropy Probe (WMAP) values for $\sigma_{P}$ and $\theta_{\text {fwhm }}$, we find for the $T B$ estimator $\sigma_{\alpha_{L M}}=7^{\circ}$ for $L=2$ [19], consistent with the current WMAP $1 \sigma$ constraint to a uniform rotation (recalling that $\alpha_{L M}=$ $\sqrt{4 \pi} \alpha$ for $L=0$ ). This then increases, by about $50 \%$, to $L=100$. At higher $L$, the noise increases due to WMAP's finite angular resolution. For WMAP, the values for $\sigma_{\alpha_{L M}}$ for the $E B$ estimator are 7 times larger and thus not constraining. Using values for $\sigma_{P}$ and $\theta_{\text {fwhm }}$ appropriate for the Planck satellite, we find that the errors to the $E B$ and $T B$ estimators are more comparable, e.g., $\sigma_{\alpha_{L M}}=1.6^{\circ}$ and $1.3^{\circ}$ for $E B$ and $T B$, respectively, for $L=2$ rising slowly to $\sigma_{\alpha_{L M}}=3.4^{\circ}$ and $1.9^{\circ}$ for $L=500$. The noise then increases rapidly for $l \geqslant 500$, when the correlation angle becomes smaller than the polarization correlation angle.
More detailed and comprehensive numerical results will be presented in Ref. [19].

I thank A. Cooray, C. Hirata, and V. Gluscevic for useful comments. This work was supported by DoE Grant No. DE-FG03-92-ER40701.

[1] M. Kamionkowski, A. Kosowsky, and A. Stebbins, Phys. Rev. D 55, 7368 (1997); M. Zaldarriaga and U. Seljak, Phys. Rev. D 55, 1830 (1997).

[2] M. Kamionkowski, A. Kosowsky, and A. Stebbins, Phys. Rev. Lett. 78, 2058 (1997); U. Seljak and M. Zaldarriaga, Phys. Rev. Lett. 78, 2054 (1997).

[3] J. Bock et al., arXiv:astro-ph/0604101.

[4] M. Zaldarriaga and U. Seljak, Phys. Rev. D 58, 023003 (1998).

[5] K. M. Smith, O. Zahn, and O. Dore, Phys. Rev. D 76, 043510 (2007); C. M. Hirata et al., Phys. Rev. D 78, 043520 (2008).

[6] A. Lue, L. M. Wang, and M. Kamionkowski, Phys. Rev. Lett. 83, 1506 (1999); N. F. Lepora, arXiv:gr-qc/9812077.

[7] S. M. Carroll, Phys. Rev. Lett. 81, 3067 (1998).

[8] B. Feng et al., Phys. Rev. Lett. 96, 221302 (2006); T. Kahniashvili, R. Durrer, and Y. Maravin, Phys. Rev. D 78, 123009 (2008); J. Q. Xia et al., Astrophys. J. 679, L61 (2008); J. Q. Xia et al., Astron. Astrophys. 483, 715 (2008).

[9] E. Komatsu et al. (WMAP Collaboration), Astrophys. J. Suppl. Ser. 180, 330 (2009).

[10] M. Pospelov, A. Ritz, and C. Skordis, arXiv:0808.0673; M. Li and X. Zhang, Phys. Rev. D 78, 103516 (2008);

[11] S. Gardner, Phys. Rev. Lett. 100, 041303 (2008).

[12] A. Kosowsky and A. Loeb, Astrophys. J. 469, 1 (1996).

[13] U. Seljak and M. Zaldarriaga, Phys. Rev. Lett. 82, 2636 (1999); M. Zaldarriaga and U. Seljak, Phys. Rev. D 59, 123507 (1999); W. Hu, Phys. Rev. D 62, 043007 (2000); T. Okamoto and W. Hu, Phys. Rev. D 67, 083002 (2003); W. $\mathrm{Hu}$ and T. Okamoto, Astrophys. J. 574, 566 (2002); M. Kesden, A. Cooray, and M. Kamionkowski, Phys. Rev. Lett. 89, 011304 (2002); L. Knox and Y. S. Song, Phys. Rev. Lett. 89, 011303 (2002); M. H. Kesden, A. Cooray, and M. Kamionkowski, Phys. Rev. D 67, 123507 (2003); A. Lewis and A. Challinor, Phys. Rep. 429, 1 (2006).

[14] A. R. Pullen and M. Kamionkowski, Phys. Rev. D 76, 103529 (2007).

[15] C. Scoccola, D. Harari, and S. Mollerach, Phys. Rev. D 70, 063003 (2004).

[16] A. Hajian and T. Souradeep, arXiv:astro-ph/0501001.

[17] W. Hu, M. M.Hedman, and M. Zaldarriaga, Phys. Rev. D 67, 043004 (2003).

[18] C. M. Hirata and U. Seljak, Phys. Rev. D 67, 043001 (2003); C. M. Hirata and U. Seljak, Phys. Rev. D 68, 083002 (2003);

[19] V. Gluscevic, A. Cooray, and M. Kamionkowski (to be published). 